\title{
Article \\ A Multi-Tool Analysis to Assess the Effectiveness of Passive Ice Protection Materials to Assist Rotorcraft Manual De-Icing
}

\author{
Jean-Denis Brassard ${ }^{1, *}$, Dany Posteraro ${ }^{2}{ }^{\mathbb{D}}$, Sarah Sobhani ${ }^{1}$, Marco Ruggi ${ }^{2}$ and Gelareh Momen ${ }^{1}$ \\ 1 Department of Applied Sciences, Univeristé du Québec à Chicoutimi (UQAC), 555 Boulevard de l'Université, \\ Chicoutimi, QC G7H 2B1, Canada; sarah.sobhani1@uqac.ca (S.S.); gelareh_momen@uqac.ca (G.M.) \\ 2 APS Aviation Inc., Groupe RHEA, 6700 Chemin de la Côte-de-Liesse, Suite 102, \\ Montréal, QC H4T 2B5, Canada; dposteraro@apsaviation.ca (D.P.); mruggi@apsaviation.ca (M.R.) \\ * Correspondence: jean-denis1_brassard@uqac.ca
}

Featured Application: A comprehensive procedure to evaluate the effectiveness of a polymeric material that aims to reduce ice and snow adhesion.

\begin{abstract}
Search and rescue missions using rotorcrafts need to be reliable all year long, even in winter conditions. In some cases of deployment prior to take off, the crew may need to manually remove accumulated contaminant from the critical surfaces using tools at their disposal. However, icy contaminant may be hard to remove since the rotorcrafts critical surfaces could be cooler than the environment, thus promoting adhesion. Currently, there exists several passive ice protection materials that could reduce the ice adhesion strength and assist the manual de-icing. The aim of this paper is to propose a detailed comparative procedure to assess the ability of materials to assist the manual de-icing of rotorcrafts. The proposed procedure consists of the characterization of materials using several laboratory tests in order to determine their characteristics pertaining to wettability, their icephobic behavior, and finally their assessment under a multi-tool analysis to evaluate if they can assist. The multi-tool analysis uses different mechanical tools, which are currently used during normal operation, to execute a gradual de-icing procedure, which begins with the softest to the hardest tool using a constant number of passes or strokes, under different types of simulated precipitation. Five different materials were used to evaluate the proposed procedure: Aluminum (used as a reference), two silicone-based coatings (Nusil and SurfEllent), an epoxy-based coating (Wearlon), and finally a commercial ski wax (Swix). All of the tested materials could assist the manual de-icing, within a certain limit, when compared to the bare aluminum. However, SurfEllent was the material that obtained the best overall results. This procedure could be easily adapted to different fields of application and could be used as a development tool for the optimization and the assessment of new materials aimed to reduce ice adhesion.
\end{abstract}

Received: 5 November 202

Accepted: 8 December 2021

Published: 13 December 2021

Publisher's Note: MDPI stays neutral with regard to jurisdictional claims in published maps and institutional affiliations.

Keywords: icephobic; de-icing; anti-icing; multi-tool; rotorcraft; coating; polymers; passive ice protection

\section{Introduction}

Search and rescue missions can be hazardous and dangerous for emergency teams, especially when winter conditions bring about snowy, stormy, or icy precipitation that adheres to emergency vehicles. The situation becomes even more difficult when rescue teams need to use aircrafts such as helicopters. If a life is in danger, the operation becomes a critical mission; therefore, rotorcraft need to be dispatched, takeoff, and land safely in any condition to successfully complete their mission. De-icing at the base is normally easier with appropriate tools, or if the rotorcraft is kept inside a hangar, icing challenges can be minimized [1]. However, the major issues may occur when they are deployed on a mission. Sometimes, ice or wet snow can deposit and adhere quickly on the critical surfaces, and it needs to be removed before an eventual takeoff. This becomes especially limiting when 
rotorcrafts are deployed to remote locations where de-icing capabilities are limited, and the crew need to rely on primarily manual methods (such as brushes or brooms) to remove snow and ice.

In a subfreezing environment, ice in the form of rime and glaze may accumulate on the surface of rotorcraft blades [2]. Rotor blades covered with a thin ice layer can have a strong negative impact on aerodynamics. Increases in drag and flow separation, which significantly reduces lift and can often make controlling the aircraft extremely difficult [3-8]. Therefore, designing methodologies that prevent ice accumulation, reduce its adhesion, or even assist during the de-icing are incredibly important in eliminating the latter issues [2]. The following sections will review the main methods presently used for this application.

Innovative de-icing and anti-icing protection systems have been developed and studied by numerous researchers. Several of these methods have also been investigated regarding their effectiveness in a laboratory setting prior to deployment in actual operations. The two main methodologies that have been explored are the following:

- Active methods; and

- Passive methods.

Active methods are those requiring external energy sources to work; they are divided in two main categories. The thermal methods consist of increasing the temperature of a critical surface to which ice may have formed or adhered. This is done to reduce ice adherence, by creating a water layer between the ice and the surface, and consequently allows for its removal under the effect of gravity. Some of these methods can also be used as a form of anti-icing. By increasing the temperature of the critical surface, it can effectively prevent supercooled water droplets from freezing upon impact. Some rotorcrafts are equipped with an electrothermal ice protection system for the main rotor blade, which can prevent grounding even in inclement icing conditions. Unfortunately, due to the significant energy load necessary for heating, the high electrical current in the slip rings, and the extensive cabling needed, approved electro-thermal de-icing systems can currently only equip the primary rotors of large rotorcrafts. However, this de/anti-icing methodology requires a significant amount of energy and adds a considerable amount of weight to the aircraft. In addition, it does not allow for the complete de-icing of the rotorcraft $[5,7,8]$.

The mechanical methods consist of using tools that break the ice adhering to the aircraft structure by shocking or inducing vibrations [7,9-12]. In most cases, mechanical methods can be considered as de-icing methods since they are used to speed the shedding process after snow or ice has accumulated or formed on the structure. Studies show that mechanical methods require approximately 100,000 times less energy than thermal methods to induce ice shedding [13].

The principal mechanical methods are based on two strategies:

- One consists of breaking the ice by means of scraping, rollers, hooks, cutters or rope; and

- The other consists of releasing energy from shock waves or vibrations to break and remove the ice.

Since ice is a very brittle material at high-strain rates, relatively little mechanical energy is required for breaking ice by using mechanical shocks as energy is not readily dissipated [7]. A main advantage of using a mechanical method instead of a thermal method is the relative ease of application. In fact, mechanical methods are the preferred choice for a timely and fast intervention to de-ice short critical sections [7]. Palacios et al. presented a novel pneumatic method to assist rotorcraft de-icing [10]. The pneumatic method consists of using a low-power, non-thermal, ultrasonic de-icing system as a potential replacement to current electro-thermal systems on helicopter rotor blades. Villeneuve et al. also proposed a low-energy de-icing system based on piezo-electric actuators [7]. The developed model allows for the design of a proper actuator to efficiently deice.

Passive methods do not use conventional sources of energy as other methods do. Instead, they use natural forces such as wind, gravity, or solar radiation. Consequently, 
they can be applied on any structure, including rotorcraft. The main passive method consists of utilizing coatings that reduce the ice adhesion on structures of interest. These coatings are known as icephobic materials and have been used in several fields [14-16]. The benefit of using icephobic coatings is the decrease in ice adhesion to a structure and/or prevent supercooled water droplets from freezing.

In order to achieve an icephobic surface, three different approaches have been introduced including fabricating a superhydrophobic surface [17], delaying ice nucleation [18], and designing a surface with low ice adhesion strength [19]. Superhydrophobic materials were introduced by Saito [20] and claimed to exhibit promising anti-icing performance. However, Saito showed in another study that by increasing the surface roughness of a superhydrophobic surface, ice adhesion increases [20]. In some cases, icephobic coatings have shown to exhibit good performance for rotor blades. Unfortunately, in most cases, these coatings lose their icephobic properties soon after application and exhibit poor durability [21,22]. Hence, research in the field of icephobic coatings for rotorcraft applications is still ongoing.

Many studies have been performed to both quantify and qualify the performance of coatings. For example, by assessing the icephobicity of superhydrophobic coatings of a helicopter blade in an icing chamber, Tarquini [23] revealed that temperature and surface roughness have a strong impact on ice adhesion. The latter study showed that by decreasing the chamber temperature and increasing the coating roughness, ice adhesion strength increases. In another study, Kimura [24] developed an icephobic coating based on acrylic urethane resin and polytetrafluoroethylene (PTFE) particles for aircraft applications. The coating proved to be superhydrophobic and effective in anti-/de-icing aircrafts.

Karmouch [25], in 2009, impregnated a PTFE nanoparticle coating on a wind turbine blade surface. It was found that the very simple dipping provided a superhydrophobic surface and reduced ice adhesion. Antonini [26] investigated the effectiveness of superhydrophobic coatings on surfaces exposed to icing conditions equipped with an electrical heater. The results showed that superhydrophobic coatings could lead to a remarkable reduction of heating power on surfaces exposed to icing conditions. Recent advances in the development of icephobic coatings were also undertaken using superhydrophobic surfaces [27-29].

In order to evaluate the capability of a system subjected to icing conditions, ice adhesion is the main parameter used in industrial and academic investigations. As a complex physicochemical process, ice adhesion is not easy to quantify or compare since it depends on process parameters. Examples of these include ice density, ice formation conditions and substrate nature, and surface profile [30]. Therefore, ice adhesion comparative tests should be performed using the same conditions throughout the evaluation process.

As discussed, many methods are available to deice rotorcrafts. Unfortunately, none of the strategies proposed is sufficient to completely prevent ice and snow accumulation. However, by combining some of these methods, for example, the thermal and coating methods, the efficiency can be significantly increased. Nevertheless, this may necessitate the deployment of complex apparatuses, which can be costly. One way to overcome this is by associating the icephobic coating with simple manual de-icing tools. This can serve as a more economical solution since it only requires the action of crew members. In fact, icephobic coatings may facilitate or assist the manual de-icing process by reducing ice adhesion. The aim of this paper is, therefore, to present a comprehensive manual de-icing procedure that will aid in assessing the effectiveness of different passive ice protection materials.

\section{Materials and Methods}

The proposed methodology consists of a multi-criterion assessment of the materials using conventional and newly developed laboratory tests. The idea is to obtain comparative results between tests so that a full assessment can be done on each coating. These tests include contact angle measurements, centrifuge ice adhesion, ice push-off, freezing delay measurements, and finally, one test that simulates operational conditions-the multi-tools analysis test. The next sections describe all tests that were carried out for this analysis. 


\subsection{Substrates}

Testing was conducted using coated and uncoated aerospace-grade 6061-T6 aluminum test plates. Each coated plate contained the specific material to be tested, whereas the uncoated plates represented the baseline or reference. This aluminum alloy was selected as it is widely used in the aerospace industry and used as the standard in previously completed studies. The characterization phase was performed using $2.5 \mathrm{~cm}$ per $2.5 \mathrm{~cm}$ substrates. The same size substrates were used during the push-off test and the freezing delay measurement. The centrifuge ice adhesion measurements were then carried out using $3.3 \mathrm{~cm}$ per $30 \mathrm{~cm}$ substrates. Finally, 30 by $50 \mathrm{~cm}$ plates were used for the multi-tools analysis.

Throughout testing, all results were compared to the aluminum baseline. Since bare aluminum reacts similarly to painted plates, the same paints as used on rotorcrafts, it was determined that these tests can be used to deduce the coating efficiency in assisted manual de-icing.

\subsection{Physical Characterization Using Contact Angle Measurement}

The first test undertaken considered the wettability of the surface using the water droplet contact angle (WCA) and contact angle hysteresis (CAH). The measurements were carried out using sessile drop technique by means of Kruss ${ }^{\mathrm{TM}}$ DSA100 goniometer at $25 \pm 0.5^{\circ} \mathrm{C}$. A $4 \mu \mathrm{L}$ water droplet was placed on the surface to evaluate the WCA by Young-Laplace approximation. By displacing the needle tip within the water droplet, the $\mathrm{CAH}$ was determined by evaluating the difference between the advancing and receding contact angles.

\subsection{Icephobic Behavior Assessment}

Three laboratory tests were used to evaluate the icephobic behavior of materials:

- The centrifugal force adhesion test (CAT);

- The push-off test; and

- The freezing delay test.

The CAT apparatus consists of a centrifuge developed at the Anti-Icing Material International Laboratory (AMIL) as seen in Figure 1.

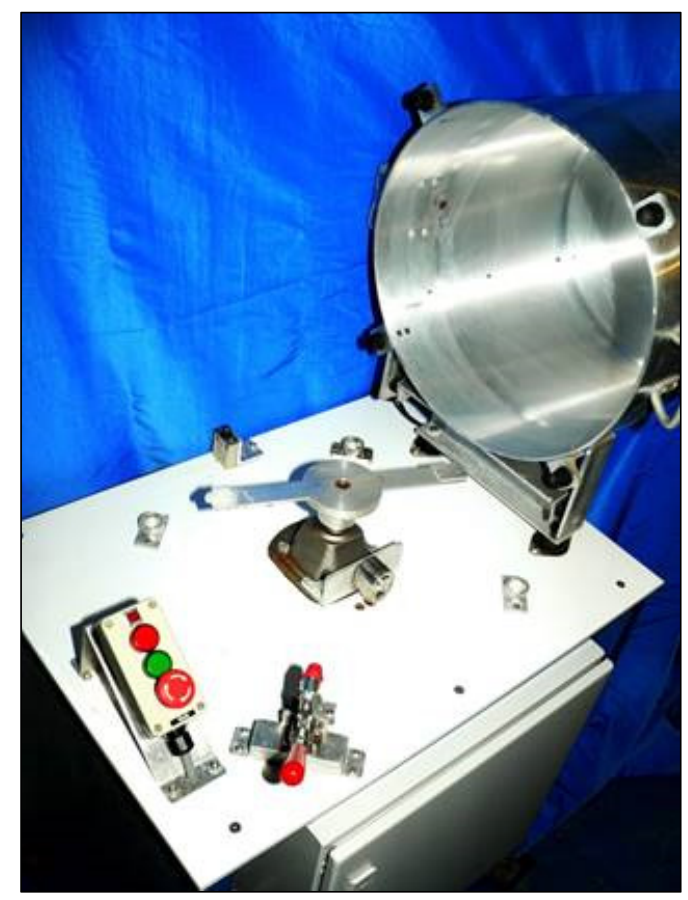

Figure 1. Overview of the centrifuge apparatus. Equipped with an accelerometer, the iced beam is rotated until the ice is detached and captured by the pan. 
The ice adhesion strength is the centrifugal shear stress exerted on the ice sample at the time of detachment. A CAT sampling consists of six different coating samples that are compared to six reference blank aluminum sheets. All samples are subjected to icing simultaneously under freezing drizzle conditions set at $-8{ }^{\circ} \mathrm{C}$ and tested at $-10.0^{\circ} \mathrm{C}$. Afterwards, samples are rotated at an acceleration speed of $300 \mathrm{rpm} / \mathrm{s}$ until the ice is detached by centrifugal force. This acceleration creates a strain rate of approximately $10^{-6} \mathrm{~s}^{-1}$. The rotational speed is controlled during the acceleration phase by the AMILmade software, which records and plots the angular velocity in real time with data from installed sensors. An accelerometer sensor located on the wall of the centrifuge is used to determine the exact time of ice detachment. This time corresponds to a sharp increase in the vibration signal. The centrifugal force is calculated using the following equation:

$$
F_{c}=m r \omega^{2}
$$

where:

$F_{c}=$ centrifugal force;

$\omega=$ ice detachment velocity;

$m=$ mass of ice; and

$r=$ beam radius at the center of the sample.

The ice adhesion shear stress is then obtained as follows:

$$
\tau_{i a}=\frac{F_{c}}{A}
$$

where:

$\tau_{i a}=$ ice adhesion shear stress;

$F_{c}=$ centrifugal force; and

$A=$ iced area in contact with the coating.

The adhesion reduction factor (ARFs) of coating $i$ is calculated as follows:

$$
A R F_{i}=\frac{\tau_{b A}}{\tau_{b i}}
$$

where:

$A R F_{i}=$ adhesion reduction factor of coating $i$;

$\tau_{b A}=$ bulk shear stress of the aluminum; and

$\tau_{b i}=$ bulk shear stress of coating $i$.

The push-off test is conducted by first placing a thin $1 \mathrm{~cm}$ diameter cylindrical plastic mold onto a substrate. The mold is then filled with deionized water and placed together with the substrate into a cold chamber at $-10.0 \pm 0.2{ }^{\circ} \mathrm{C}$ for $24 \mathrm{~h}$ to form an ice cylinder. The test sample is placed onto the holder and fixed into position by a vacuum (using two specialty screws on the apparatus). In our test, a remote computer-controlled interface controlled the velocity of these specialty screws at a fixed rate of $0.05 \mathrm{~mm} \cdot \mathrm{s}^{-1}$ so that the sample holder gradually approached the force gauge. The force gauge was set to measure the shear force every Deci-second (one-tenth of a second) until the ice was detached. The adhesion stress was then calculated based on the maximum force and icing area. As previously stated, the adhesion reduction factors (ARF) of the coatings are obtained by dividing the bulk shear stress value of the uncoated aluminum sheets by the bulk shear stress of the coated sheets. Figure 2 illustrates the push-off apparatus.

The two methods previously described were used to determine ice adhesion. The CAT is a method that uses simulated atmospheric ice, while the push-off test is a better representative of ice removal during actual operations. The use of the centrifugal force, however, allows for a direct comparison of coatings. 


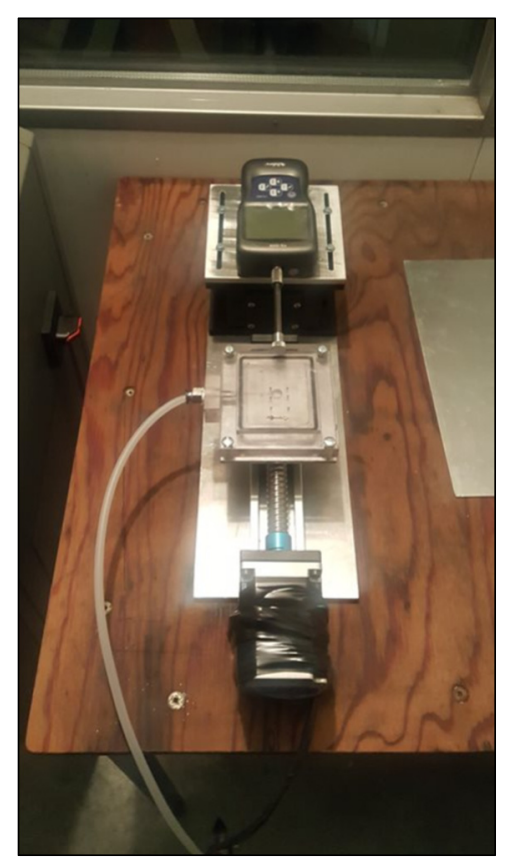

Figure 2. Overview of the push-off apparatus. The ice adhering to the coating is pushed off by the force gauge and evaluates the adhesion.

The final method consists of measuring the water freezing delay on the coatings interface, namely the nucleation time. The experimental freezing device used allows for the precise determination of the time required for a water drop to solidify under controlled environmental conditions. The volume of the water droplet was $10 \mu \mathrm{L}$ and the test was performed at $-10^{\circ} \mathrm{C}$. The reported values are the average of seven experimental runs.

These three laboratory tests were used to predict the icephobic behavior. In the case where more materials need to be evaluated, these methods could be used for screening purposes to adequately select the most promising materials prior to performing an extended evaluation.

\subsection{Multi-Tools Analysis}

The goal of this study was to evaluate coatings under specific environmental conditions that simulate actual operations. For this purpose, a procedure based on utilizing different tools under different icing conditions was developed. The test protocol is based on the Mohs scale. The Mohs scale was invented in 1812 by the German mineralogist Friedrich Mohs to measure the hardness of minerals. It is based on 10 readily available minerals. As it is an ordinal scale, one must proceed by comparison (ability of one to scratch the other) with two other minerals of known hardness. This scale is neither linear nor logarithmic.

For this project, the comparison scale uses the five tools as illustrated in Figure 3. These tools are available for purchase at any local supply store. All tools were classified in order of hardness according to the expertise of the research team.

In Figure 3, tool A represents a typical car brush. The brush is generally the first piece of equipment used to remove snow or other loose contamination. The second tool, shown in Figure 3 (tool B), is the flexible polymer blade. This type of instrument allows for the removal of snow without damaging the surface. For the purpose of this study, only the widest part was used. The third tool utilized, tool C, was a squeegee as depicted in Figure 3. Normally used for window cleaning, the squeegee was slightly stronger than the flexible polymer blade but less than the hard polymer blade (tool D in Figure 3). Lastly, two rigid polymer blades, one smooth and one corrugated (tool D and tool E in Figure 3, respectively), were used for de-icing. Both blades are normally used to remove adhering contaminants from surfaces and require the application of some form of pressure and, most importantly, the passage of several steps for complete removal. 


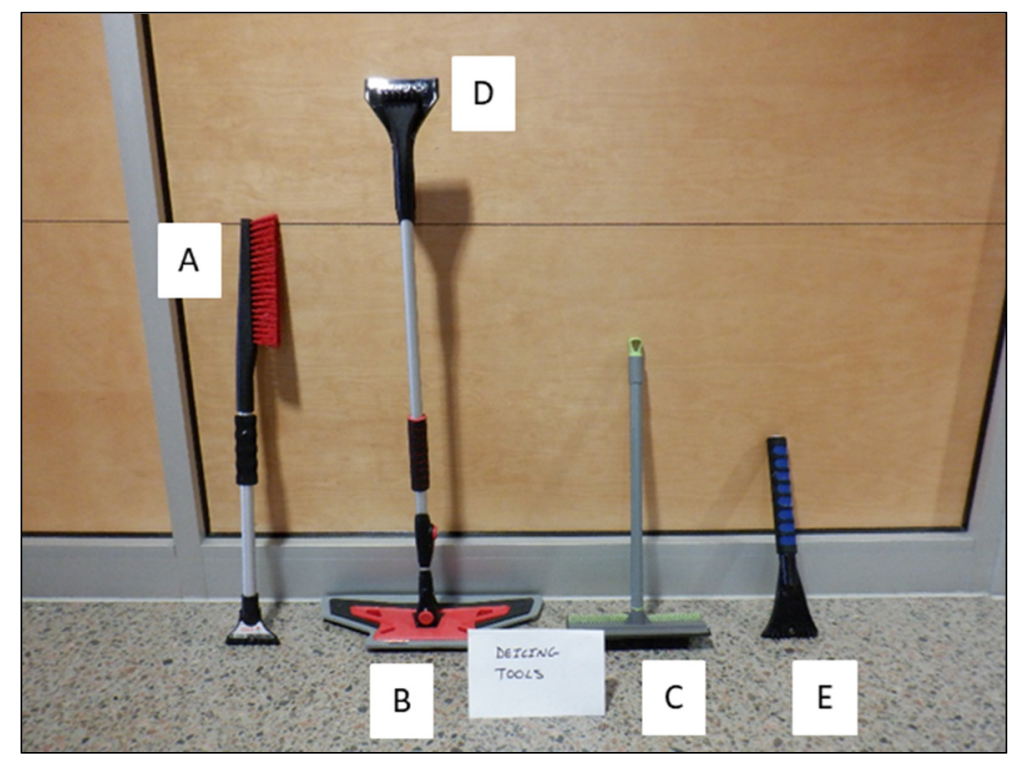

Figure 3. De-icing tools used in the multi-tools analysis, which range from softest to hardest: (A) the brush, (B) the flexible polymer blade, (C) the squeegee, (D) the hard polymer blade, and (E) the corrugated polymer blade.

The protocol was to begin with the brush (least amount of force needed during deicing) and conduct five passes (sweeps). If contamination was still present, we moved on to the next available tool with respect to increasing force (flexible polymer blade), and repeated until all contamination was removed or until all tools were tested with five passes. The objective was to identify the level of force and number of passes required to remove the ice. These icing and de-icing tests were performed in the AMIL 9M cold room. All de-icing tests were performed by the same technician and in the same timeframe to ensure repeatability. The technician also applied the same pressure on each material during each icing condition. The speed of passing each tool, the positioning of hands on each tool, and the technician's posture and stance, along with plate restraining, were all kept consistent. After each passage, the plates were inspected visually, photographed, and weighed.

\subsection{Icing Conditions}

The above-mentioned tools were used under four different icing conditions to evaluated how each coating may assist in manual de-icing.

The first icing condition consisted of high-intensity freezing rain (HZR) of short duration at a temperature of $-10^{\circ} \mathrm{C}$. For operations, this type of precipitation can occur in a very short period of time and coat cold rotorcraft surfaces with a thin layer of ice. The duration of precipitation was determined to be $5 \mathrm{~min}$. This precipitation was generated using two $1000 \mu \mathrm{m}$ pneumatic sprinklers with tap water at a temperature of $4{ }^{\circ} \mathrm{C}$ positioned at the top of the cold chamber. The total water droplet free fall distance was $7.1 \mathrm{~m}$. The rate of precipitation was calibrated by adjusting the "On" time and "Off" time of the sprinkler. The sprayers were kept centered with the available support. A typical representation of the obtained icing condition is presented in Figure 4. The resulting ice does not cover the entire surface but is interconnected, and the contaminant adheres strongly to the substrates. 


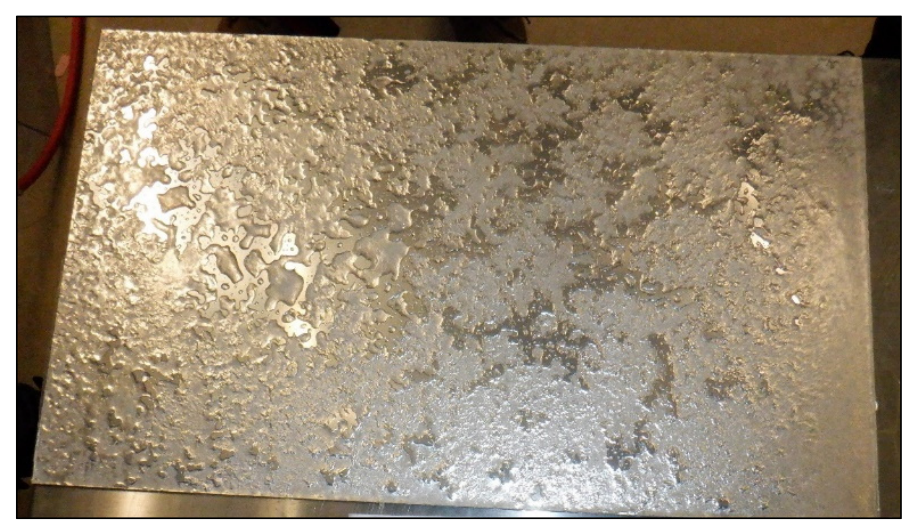

Figure 4. High-intensity freezing rain over aluminum reference plate.

The second condition consisted of low-intensity freezing rain (LZR). The lower icing intensity was obtained using the same conditions as the high-intensity freezing rain but with the application of an "Off" time of $25 \mathrm{~s}$ for about $2 \mathrm{~min}$. Under these conditions, the performance of the hydrophobic coated surfaces was exploited, to a greater extent, allowing for the formation of ice beads instead of an ice sheet. The amount of ice obtained on the plate was not as uniform as the high-intensity freezing rain and is seen in Figure 5.

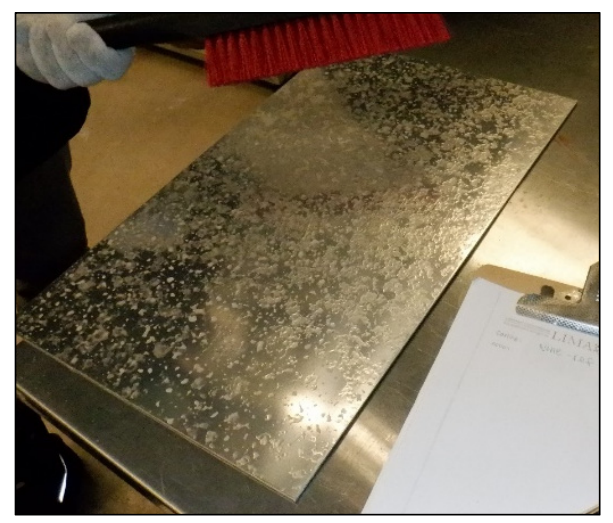

Figure 5. Low-intensity freezing rain over aluminum reference plate.

The third condition consisted of dry snow. This was done to simulate an aircraft, which landed prior to a dry snow event and was cold soaked. The snow used during testing was obtained from a snow event that occurred 3 days prior to testing. This snow was collected in a cooler and stored in a freezer at a temperature of $-10^{\circ} \mathrm{C}$. Prior to testing, a $200 \mathrm{~mm}$ diameter sieve with $1.4 \mathrm{~mm}$ openings was used to sift the snow onto the test plate. The average snow accumulation for each test was $3 \mathrm{~mm}$ as seen in Figure 6 .

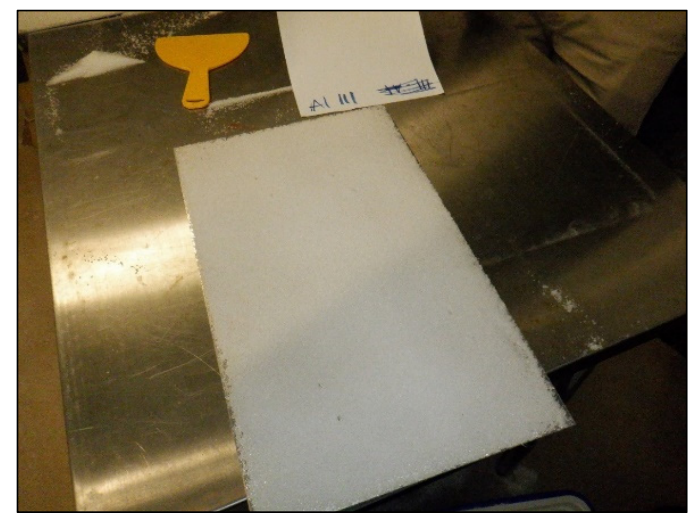

Figure 6. Dry snow over aluminum reference plate. 
The fourth condition consisted of partially melted and refrozen snow. To simulate a more conservative or worse condition involving snow, the accretion of snow was performed on a warm surface in a cold room. In an operational perspective, this simulates an aircraft, which landed during a dry snow event with some surfaces above the freeze point (warm soaked surface), likely due to engine exhaust, etc. This was achieved by sifting snow as previously described for dry snow, with the aluminum plate tempered to $8{ }^{\circ} \mathrm{C}$ and subsequently placed in the cold chamber at $-10{ }^{\circ} \mathrm{C}$ for the application of snow. As the snow was accumulating on the test plate, the initial snow particles melted and refroze with the cooling of the plate, and snow was added until a $3 \mathrm{~mm}$ thickness was achieved. The result was snow and ice adhesion on the test plate with added snow on the surface as shown in Figure 7.

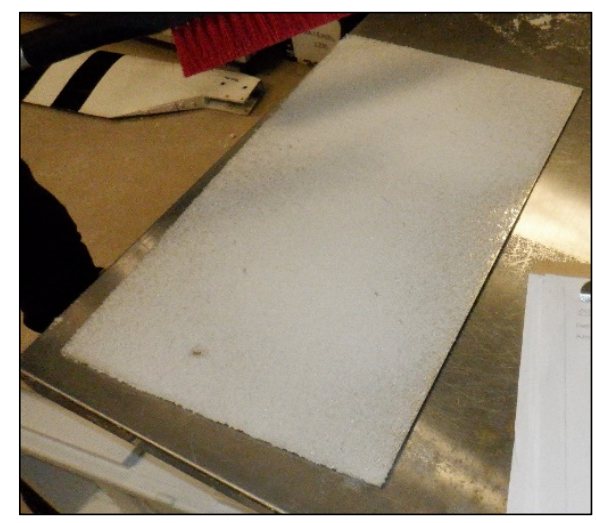

Figure 7. Partially melted and refrozen snow over aluminum reference plate of $30 \mathrm{~cm}$ by $50 \mathrm{~cm}$.

\subsection{Materials}

Five different materials were used to evaluate the proposed procedure and to assess their efficiency in assisting a manual de-icing. The test materials were as follows:

- Aerospace-grade 6061-T6 aluminum

As previously stated, the aluminum test plate served as the benchmark or reference. The surface finish as well as its wettability can thus be used to represent a painted rotorcraft surface.

- $\quad$ Nusil

This material consisted of a commercial silicone elastomer named Nusil. A hydrophobic surface results when applied to different substrates. The silicone polymerizes at room temperature to obtain a smooth surface finish. A primer was added to the surface prior to application to promote silicone adhesion. Since silicone is a viscous material prior to polymerization, it was applied using a simple brush.

\section{- $\quad$ SWIX LF5}

This material consisted of a commercial Ski wax named SWIX LF5. This material is fragile when applied to a substrate and is generally used to enhance the performance of skiers in alpine ski. It is a fluoro-carbon-based wax, which is most effective in the temperature range of $-8{ }^{\circ} \mathrm{C}$ to $-14^{\circ} \mathrm{C}$. Fluorinated components have been shown to be efficient against water and ice adhesion. The application process consisted of heating the wax to $150{ }^{\circ} \mathrm{C}$ using an electric iron. Once melted, it was applied uniformly on the substrates which were at room temperature.

\section{- SurfEllent}

This material consisted of the commercial coating named SurfEllent. It is a commercially advanced anti-icing coating and is used for multiple applications. The material formulation is based on polydimethylsiloxane and acetoxy silane cross-linker. The coating was applied using a spray applicator. 


\section{- Wearlon super F1-Ice}

This material is the commercial coating named Wearlon super F1-Ice. It is an epoxy/ latex-based coating used on communication towers and bridge structures. The coating consists of two-parts applied simultaneously by a spray applicator.

The selection of different materials that are based on differing chemical compositions allows for a comprehensive assessment of the proposed procedure. Each material consisted of a thin and uniform layer that entirely covered the surface.

\subsection{Scoring Methodology}

The scoring methodology employed consists of ranking each result with a maximum score of 85 .

The score of the CAT, push-off test, and freezing delay were based on the value of the reduction factor compared to that of the reference. In order to adequately score the performance of all materials tested, both the ARF and MTA were used. It is important to note that the MTA should have a greater weight on the final score compared to the ARF due to its significance in real-world operations. Therefore, in order to decrease the impact of the ARF values on the final results and increase the impact of the MTA, the ARF values were proportionally down scaled using a maximum value of 5 . The surface with the best ARF thus obtains a value of 5, while the others are reduced on the scale. This new score out of 5 was named the icephobic rating.

For the MTA tests, the maximum possible score of 20 represents the most effective de-icing process, which consists of using the softest tool, i.e., the brush, and eliminates all contaminants with a single pass. A score of 0 thus represents the most ineffective de-icing process and resulted in the use of the hardest coarse polymer blade with 5 consecutive passes. The latter score is obtained if contamination is still present after the de-icing process is complete. Thus, the maximum score per condition is 20 with a maximum result of 80 .

\section{Results and Discussion}

The results presented in this paper are as follows: the characterization results, the icephobic behavior, and the MTA results. The characterization results are presented in Table 1.

Table 1. Wettability results.

\begin{tabular}{cccccc}
\hline Material \# & 1: Reference & 2: Nusil & 3: Swix & 4: SurfEllent & 5: Wearlon \\
\hline Material Type & Aluminum & RTV Silicone & Fluorinated wax & Silicone Coating & Epoxy coating \\
CA $\left(^{\circ}\right)$ & $93 \pm 2$ & $114 \pm 2$ & $112 \pm 1$ & $112 \pm 2$ & $110 \pm 3$ \\
CAH $\left({ }^{\circ}\right)$ & $34 \pm 3$ & $6 \pm 2$ & $6 \pm 2$ & $10 \pm 1$ & $35 \pm 3$ \\
\hline
\end{tabular}

The procedure consisted of two main comparisons. The first was between the experimental materials and the reference aluminum, while the second was between the materials themselves. This allowed us to draw two main conclusions: (i) to determine if the proposed material was better than that of the reference and (ii) to establish which product was best for each condition, which in turn allowed for its classification. The characterization of the material was done using contact angle and contact angle hysteresis, which gave relevant information to understanding their behavior under the precipitation. The reference material, aluminum, had a contact angle of $93 \pm 2^{\circ}$. The surface was at the limit of being considered hydrophobic. However, the contact angle hysteresis, measured at $34 \pm 3^{\circ}$, shows that the surface was not at all uniform and that it could have some asperities that could aid in anchoring water. When a layer of material \#2, i.e., Nusil, was applied to the same aluminum, the contact angle increased to $114 \pm 2^{\circ}$, signifying that the surface is clearly hydrophobic. Since the layer of the RTV silicone was sufficient to fill all of the aluminum's asperities, the contact angle hysteresis was then reduced to a value of $6 \pm 2^{\circ}$. Visually, the Nusil RTV silicone is soft and smooth. In the same way, the Swix Ski Wax, since it is more friable, showed results comparable to that of Nusil, with a contact angle of 
$112 \pm 1^{\circ}$ and a contact angle hysteresis of $6 \pm 2^{\circ}$. The SurfEllent silicone coating also had a contact angle in the same range with a value of $112 \pm 2^{\circ}$ but with a slightly higher contact angle hysteresis of $10 \pm 1^{\circ}$. Finally, material \#5, i.e., the epoxy-based coating Wearlon, had a contact angle in the same range with a value of $110 \pm 3^{\circ}$; however, its contact angle hysteresis was practically the same as that of aluminum with a value of $35 \pm 3^{\circ}$. From these results, we can see that all of the materials' outer surfaces are in the same range with respect to contact angle and are more hydrophobic than that of the reference material, aluminum.

The same comparisons can be made with the icephobic behavior characteristics as presented in Table 2.

Table 2. Icephobic assessment.

\begin{tabular}{cccccc}
\hline Material \# & 1: Reference & 2: Nusil & 3: Swix & 4: SurfEllent & 5: Wearlon \\
\hline$\tau$ CAT (KPa) & $609.5 \pm 36.8$ & $72.5 \pm 4.1$ & $319.0 \pm 70$ & $89.2 \pm 1.9$ & $101.5 \pm 9.1$ \\
ARF CAT & - & 8.4 & 2.2 & 6.8 & 6.0 \\
$\tau$ PO (KPa) & $604.0 \pm 8.6$ & $48.5 \pm 8.5$ & $56.4 \pm 9.7$ & $67.9 \pm 4.1$ & $527.0 \pm 5.6$ \\
ARF PO & - & 12.5 & 10.7 & 8.9 & 1.1 \\
Freezing delay (sec) & 126 & 1982 & 1045 & 828 & 336 \\
\hline
\end{tabular}

The two first characteristics are concerned with the ice adhesion measurement using two laboratory tests: the centrifuge adhesion test (CAT) and the push-off test (PO). These two methods are complimentary to each other since the CAT uses simulated atmospheric ice and is widely used and recognized by the industry. While PO, although it uses molded ice, better mimics the passage of a tool, and is therefore more representative of the targeted application presented here. The results presented for both aforementioned methods are illustrated as a function of bulk shear stress $(\tau)$ and also in terms of adhesion reduction factors (ARF). The first material, i.e., the reference aluminum, had an ice adhesion value in the same range for both methods with $609.5 \pm 36.8 \mathrm{KPa}$ and $604.0 \pm 8.6 \mathrm{KPa}$ for CAT and $\mathrm{PO}$, respectively. This value of ice adhesion is in the typical range obtained for bare aluminum. The material showing the best results was Nusil with ARFs of 8.4 and 12.5 for the CAT and PO, respectively. It signifies that the ice adhesion was reduced by $88 \%$ to $97 \%$ depending on the method. The second-best ranked material was SurfEllent, with ARFs of 6.8 and 8.9 for the CAT and PO, respectively. SurfEllent, similarly to Nusil, is a silicone-based coating. Both showed a contact angle thay is clearly hydrophobic and a contact angle hysteresis below $10^{\circ}$, which shows that they are unlikely to interact with water in its liquid state or adhere with water in its solid state. The second element to consider was their uniformity and smoothness. Both offered a complete smooth coating of the surface and did not show visual anchoring points for ice adhesion. The third-best ranked material was the Swix wax with an ARF of 2.2 with the CAT and an ARF of 10.7 with the PO. The results were much better with the PO than the CAT. This can be attributed to the type of ice used during the tests. Since the waxy material is not designed to adhere perfectly to a metal surface, the coating may not be completely uniform. So, when small droplets like freezing drizzle are deposited on the materials surface, some of these droplets will remain on the top of the wax; however, some may penetrate further to the substrates surface and can thus partially anchor themselves with the aluminum. This phenomenon is less pronounced with the PO test because the ice is formed by molding a fixed quantity of water that does not penetrate the wax prior to freezing. The same reasoning can be drawn with the contact angle since the water drop of $5 \mu \mathrm{L}$ remains on the top and does not penetrate. Finally, the last performant material, in terms of low ice adhesion, was the epoxy base Wearlon. Although the result in the CAT provided an ARF of 6.0, the ARF in the PO test was only 1.1. In this particular case, we can correlate it with the value of the contact angle hysteresis, which is practically the same as that of aluminum, and thus the presence of anchoring points promotes ice adhesion. Visual inspection of the Wearlon also confirmed the presence of millimetric anchoring points that may increase ice adhesion. Materials may react differently depending on the icing conditions used. Therefore, ice adhesion 
measurements illustrate the importance of using multiple methods prior to establishing a material as icephobic.

To deepen the understanding of icephobic material behavior for aircraft application, it is necessary to thoroughly analyze the mechanism of ice formation (the process of nucleation and growth). The fundamental and crucial step for the formation of ice is known as nucleation. Freezing of a small quantity of water (e.g., a droplet) does not occur immediately after contact with a cold sub-zero surface. Prior to nucleation, the time required for a droplet of water to freeze, once in a supercooled state, is called the induction period. This induction period if often referred to as the "freezing time delay." In this project, the longer the freezing delay, the better the material. The freezing time delay is defined as the time interval between the impacting of the water droplet on the pre-cooled substrate and the onset of freezing.

Preliminary data on the freezing delay of the water droplet for the reference material surface, aluminum, were determined to be $126 \mathrm{~s}$. Overall, the initial results showed that the maximum freezing delay of $1982 \mathrm{~s}$ was achieved with the Nusil surface, which was more than 15 times that of the reference aluminum. In other words, the formation of ice can be effectively delayed by the Nusil surface at a temperature and humidity of $-10^{\circ} \mathrm{C}$ and $20 \%$, respectively. The effect was less with Swix with $1045 \mathrm{~s}$ and SurfEllent with $828 \mathrm{~s}$, but they were still 6 to 8 times longer than aluminum. Finally, the Wearlon provided the poorest performance when compared to the other materials with a freezing delay of $336 \mathrm{~s}$. The benefit obtained from these experiments is that the freezing delay can be used as a prediction tool to evaluate the effectiveness of the material. The longer the freezing delay, the longer the user has to remove the contamination before it adheres and requires more energy for removal.

\subsection{Multi-Tool Analysis}

The following section describes the results of the multi-tool analysis (MTA). Refer to Figure 3 for details related to the de-icing tools used, as they are referred to by a range of numbers in the figures of this section. Using the four icing conditions identified in Section 2.5, the plates were subjected to manual MTA de-icing cycles with a gradation in tool hardness. The results are presented in Table 3.

Table 3. MTA results.

\begin{tabular}{|c|c|c|c|c|c|}
\hline & 1: Reference & 2: Nusil & 3: Swix & 4: SurfEllent & 5: Wearlon \\
\hline $\begin{array}{l}\text { High- } \\
\text { intensity } \\
\text { freezing rain }\end{array}$ & $\begin{array}{c}\text { Score: } 0 \\
\text { Hard, ridged } \\
\text { polymer } \\
5 \text { passes } \\
\text { Ice remaining }\end{array}$ & $\begin{array}{l}\text { Score: } 6 \\
\text { Hard } \\
\text { polymer } \\
5 \text { passes }\end{array}$ & $\begin{array}{l}\text { Score: } 10 \\
\text { Hard } \\
\text { polymer } \\
1 \text { pass }\end{array}$ & $\begin{array}{c}\text { Score: } 1 \\
\text { Hard, ridged } \\
\text { polymer } \\
5 \text { passes }\end{array}$ & $\begin{array}{c}\text { Score: } 0 \\
\text { Hard, ridged } \\
\text { polymer } \\
5 \text { passes } \\
\text { Ice remaining }\end{array}$ \\
\hline $\begin{array}{l}\text { Low- } \\
\text { intensity } \\
\text { freezing rain }\end{array}$ & $\begin{array}{c}\text { Score: } 0 \\
\text { Hard poly- } \\
\text { mer } 5 \text { passes } \\
\text { Ice remaining }\end{array}$ & $\begin{array}{l}\text { Score: } 6 \\
\text { Medium } \\
\text { polymer } \\
5 \text { passes }\end{array}$ & Not tested & $\begin{array}{l}\text { Score: } 9 \\
\text { Medium } \\
\text { polymer } \\
2 \text { passes }\end{array}$ & $\begin{array}{l}\text { Score: } 9 \\
\text { Medium } \\
\text { polymer } \\
2 \text { passes }\end{array}$ \\
\hline Dry snow & $\begin{array}{c}\text { Score: } 20 \\
\text { Brush } \\
1 \text { pass }\end{array}$ & $\begin{array}{c}\text { Score: } 14 \\
\text { Soft polymer } \\
2 \text { passes }\end{array}$ & $\begin{array}{l}\text { Score: } 20 \\
\text { Brush } \\
1 \text { pass }\end{array}$ & $\begin{array}{l}\text { Score: } 20 \\
\text { Brush } \\
1 \text { pass }\end{array}$ & $\begin{array}{c}\text { Score: } 15 \\
\text { Soft polymer } \\
1 \text { pass }\end{array}$ \\
\hline $\begin{array}{l}\text { Adhering } \\
\text { snow }\end{array}$ & $\begin{array}{l}\text { Score: } 5 \\
\text { Hard } \\
\text { polymer } \\
1 \text { pass }\end{array}$ & $\begin{array}{l}\text { Score: } 5 \\
\text { Hard } \\
\text { polymer } \\
1 \text { pass }\end{array}$ & $\begin{array}{l}\text { Score: } 10 \\
\text { Medium } \\
\text { polymer } \\
1 \text { pass }\end{array}$ & $\begin{array}{l}\text { Score: } 10 \\
\text { Medium } \\
\text { polymer } \\
1 \text { pass }\end{array}$ & $\begin{array}{l}\text { Score: } 8 \\
\text { Medium } \\
\text { polymer } \\
3 \text { passes }\end{array}$ \\
\hline
\end{tabular}

The results are presented in terms of the score obtained as per the procedure explained in Section 4. To recap, the scores can range from 20, which is considered the most effective, to 0 , which is considered the least effective, as seen in red in Table 3. For simplicity, the 
tool name and the number of passes were added. Each type of precipitation is treated individually and is discussed below.

\subsubsection{MTA vs. High-Intensity ZR}

The first condition to be investigated was high-intensity freezing rain. The resulting ice, regardless of material tested, consisted of an even ice cover. The results are presented in Figure 8 as the weigh percentage of remaining ice contaminant, as a function of tool used, and as the number of passages conducted. When the mass remains constant, it indicates that the tool passed over the ice without removing any. For all coatings except the ski wax, the use of the brush (A) and the soft polymer blade (B) did not result in any ice removal. In the specific case of the ski wax, the first pass of the flexible polymer blade (B) resulted in the removal of approximately $25 \%$ of the mass of ice. In all cases, the use of the rigid polymer blade (D) resulted in the removal of the most significant amounts of contaminants. In some cases, the use of the ridged, hard polymer blade was necessary to complete the de-icing cycle. In two cases, namely the aluminum baseline and Wearlon, the selected tools did not completely remove the ice. The obtained results correlate well with the results obtained using the PO. For this condition, the best materials were the Ski Wax, with a score of 10, and Nusil, with a score of 6 . However, the SurfEllent, with a score of 1 , still assisted de-icing in a more effective manner than the reference surface. Generally, the de-icing process was much easier on soft materials such as Nusil; however, this type of surface finish did slightly hinder the passage of certain blades. Although the Swix provided the best results, the material was broken and torn apart during the de-icing process and acted more like a one-time use sacrificial layer.

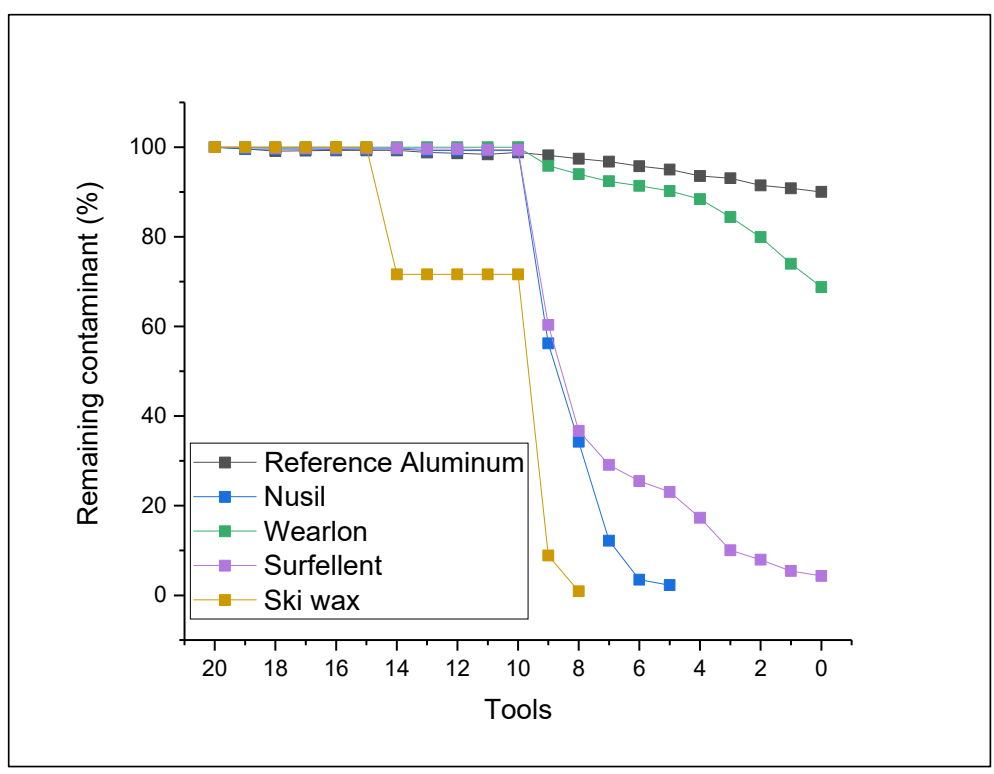

Figure 8. Multi-tool analysis under high-intensity freezing rain. Note: Numbers correspond to the tool used and number of passages during de-icing. Where "20 to 16 " represent the broom, " 15 to 11 " represent the soft polymer blade, " 10 to 6 " represent the hard polymer blade, " 5 to 1 " represent the ridged polymer blade, and 0 indicates that ice remained on the surface.

\subsubsection{MTA vs. Low-Intensity ZR}

The second type of icing simulated was low-intensity freezing rain. The results are presented in Figure 9 as the weigh percentage of remaining ice as a function of tools and the number of passages. The corresponding picture after icing is illustrated in Figure 5. At the lower intensity of freezing rain precipitation, the materials' hydrophobic properties are more effective and can therefore delay the solidification of water droplets or cause them to solidify in beaded form. In this case, an additional tool was added that was stiffer than the soft polymer blade (B) but less than the solid polymer blade (D), namely the squeegee 
(tool C). The aluminum surface was again observed to be the least favorable one, not allowing for the complete de-icing after the passage of all tools. In the case of the SurfEllent coating, however, the soft polymer blade (B) was able to partially remove some of the ice. In almost all cases, the use of tool C (squeegee) removed most of the contaminants from the Wearlon- and SurfEllent-coated surfaces. Nevertheless, the use of the solid blade was necessary to remove some residual contamination present on the Nusil surface. Note that the ski wax was not tested in this condition. However, we can assume that a similar result to the previous condition would be obtained.

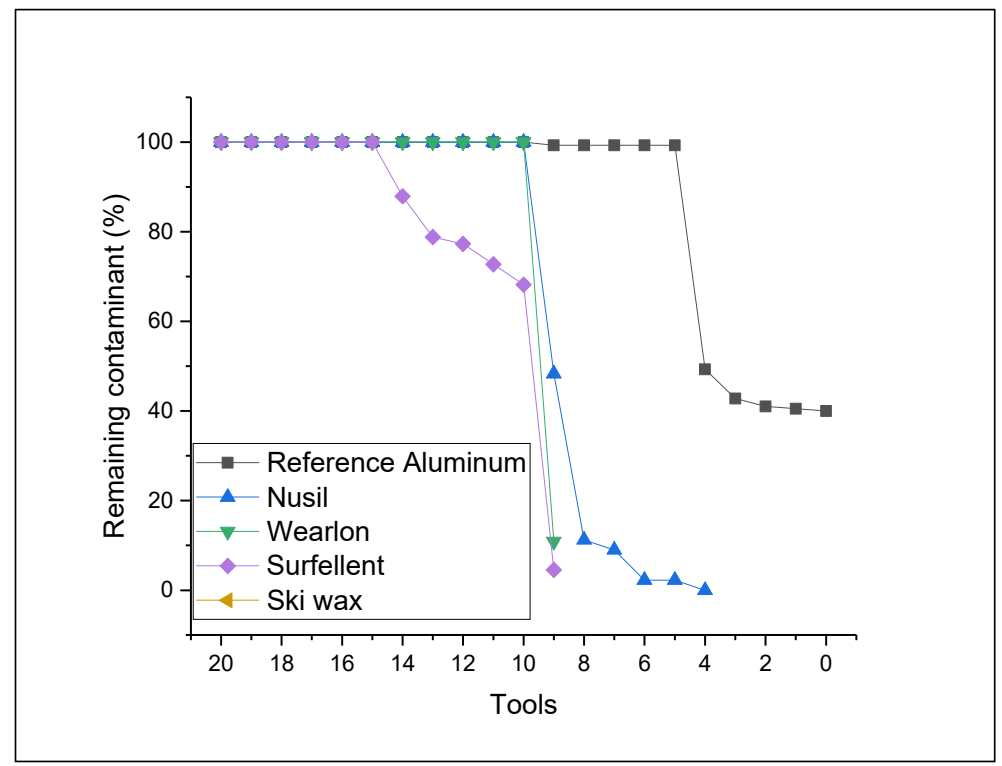

Figure 9. Multi-tool analysis under low-intensity freezing rain. Note: Numbers correspond to the tool used and the number of passages during de-icing. Where "20 to 16 " represent the Broom, " 15 to 11 " represent the soft polymer blade, " 10 to 6 " represent the squeegee, " 5 to 1 " represent the hard polymer blade, and 0 indicates that ice remained on the surface.

A simple 2D representation of ice covering two different tested materials is presented in Figure 10 below. The left portion of the figure shows how ice typically accumulates on an aluminum surface. The ice is shown to cover the surface uniformly, exploiting the available roughness and all anchoring points, which in turn increases ice adhesion. In the same manner, when the MTA is applied using soft tools, such as the broom and the polymer, they are not rigid enough to break the adhesive bond with the surface. Instead, the cohesive bonds are first broken within the ice itself followed by the breakage of adhesive bonds. Therefore, a harder tool, such as hard and ridged polymer, is needed to effectively remove the ice. In the case of hydrophobic materials, such as a Nusil coating for example, the film of ice is not uniform, as represented in the right portion of Figure 10. The higher contact angle and the longer freezing delay of the material causes the water to agglomerate in large beads (droplets) prior to freezing. This effect is more noticeable when the precipitation rate is low. However, since the surface is hydrophobic, the ice covering the surface is less uniform, which reduces the adhesion of ice compared to that of aluminum alone. Hence, the passage of a soft tool allows for the gradual removal of small pieces of ice with substantially less effort. In essence, there is no need to break the cohesive bond prior to the adhesive bond since it is weaker when compared to the reference aluminum surface. 

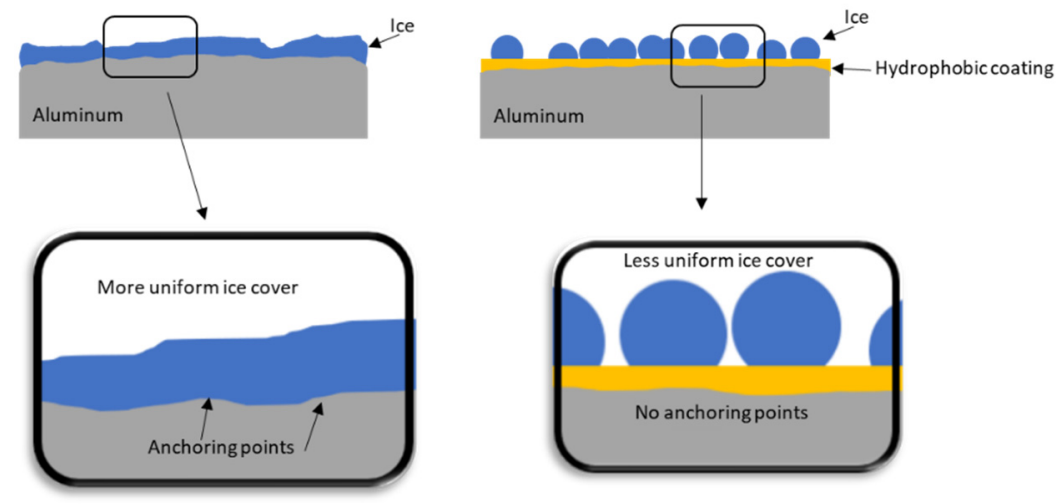

Figure 10. 2D representation of ice accumulation over bare aluminum (left portion) and over hydrophobic coating (right portion).

\subsubsection{MTA vs. Dry Snow}

The third condition tested was dry snow. This precipitation is non-adherent when falling on a cold-soaked surface, and this type of precipitation occurs very commonly in the winter season. The main results are presented in Figure 11.

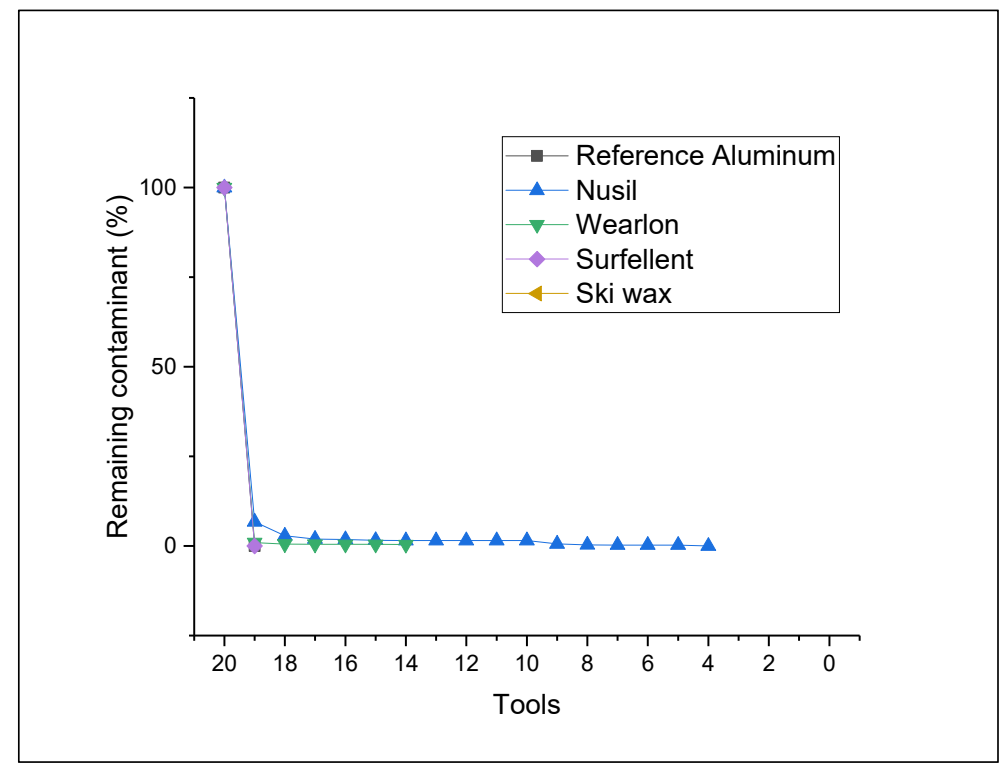

Figure 11. Multi-tool analysis under dry snow. Note: Numbers correspond to the tool used and number of passages during de-icing. Where " 20 to 16 " represent the broom, " 15 to 11 " represent the soft polymer blade, "10 to 6 " represent the squeegee, and " 6 to 1 " represent the hard polymer blade.

In almost all cases, including aluminum, the snow was removed on the first pass of the brush (the softest tool). However, two surfaces, Wearlon and Nusil, caused a very thin layer of snow to stick to their surfaces, requiring the use a rigid surface to scrape it off effectively.

It was initially expected that all materials would react the same in the presence of dry snow. However, soft materials such as Nusil allowed for a very small amount of snow to stick to its surface upon passage of the broom. By using the soft polymer, the remaining snow was easily removed. As presented in Figure 11, further passage of the tools was undertaken to ensure that all contamination was removed.

\subsubsection{MTA vs. Dry Snow on a Warm Surface}

Finally, in the fourth targeted condition, the cold snow was deposited on a warm surface having an approximate temperature of $8{ }^{\circ} \mathrm{C}$. This was done in order to replicate 
a scenario where a critical surface was heated by the warm air of an engine. The snow thus partially melted and, under the effect of the cold temperature of the chamber, refroze and adhered to the surface. The results obtained using different surfaces are shown in Figure 12 as the weigh percentage of remaining contaminant as a function of tools used and the number of passages.

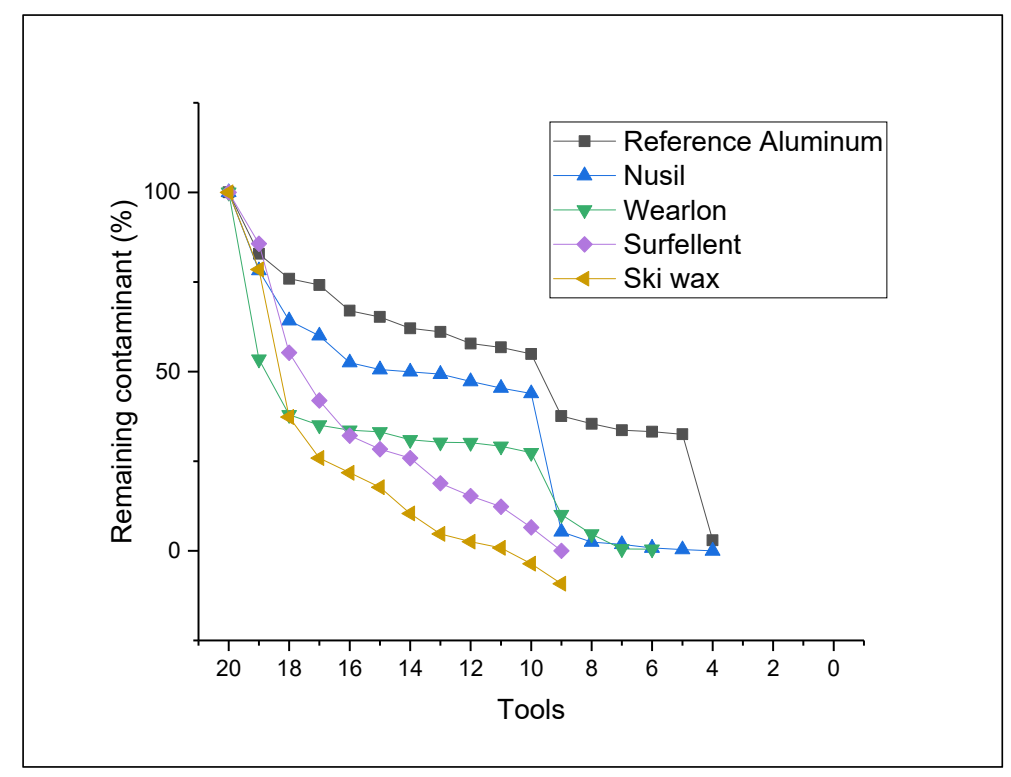

Figure 12. Multi-tool analysis under partially melted and refrozen snow-simulation of dry snow on a warm soaked surface. Note: Numbers correspond to the tool used and number of passages during de-icing. Where " 20 to 16 " represent the broom, " 15 to 11 " represent the soft polymer blade, " 10 to 6 " represent the squeegee, and " 5 to 1 " represent the hard polymer blade.

In all cases, it was possible to remove the contaminants. In the first instance, the first layer of snow that was not melted and was situated over the resolidified contamination was removed by brushing. The reference surface, aluminum, required the passage of a solid polymer blade to completely remove all surface contamination. The SurfEllent, Swix, and Wearlon were similar in terms of difficulty in removal of surface contamination. Both required tool $\mathrm{C}$, i.e., the Squeegee, to remove most of the contamination followed by the medium polymer to remove the remaining frozen snow. The Nusil coating performed similarly to the aluminum reference as contamination was mostly removed with the hard polymer blade.

Typically, this case where snow is adhering to a critical surface represents the most conservative case, which could be encountered by crew members while inflight. Because some of the snow partially melted upon contact with the warm surface, the resulting melt water can penetrate both the surface and the remaining unmelted snow and thus act as an adhesive.

\subsection{Correlating the Laboratory Results of Plates and Rotor Blade Sections}

In order to validate the procedure for use on rotorcraft tail blade profiles, it was determined that scaling upward from a plate generated correlated results. This in turn validated the procedure for use on rotorcraft tail blades. It is important to note that the goal was to determine if the military blade, painted in matte, and the commercial blade, painted in gloss (both salvaged from an operational aircraft) presented in Figure 13, would generate similar results to the aluminum baseline test plate surfaces. 


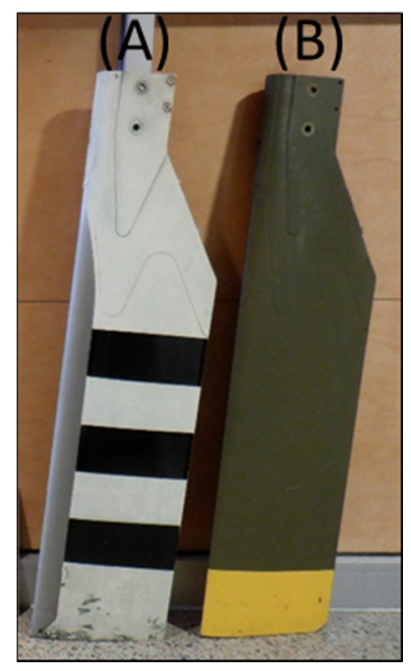

Figure 13. (A) Commercial gloss and (B) military matte-painted tail blade profiles.

All tools previously described in Sections 2.4 and 3.1 were used in the same order with the same numbers of passages. When considering the condition of HZR, for all samples tested, ice remained after all tools were used with five passes. The same results were obtained with the matte, gloss profiles, and under the condition of LZR. When tested using snow as the contamination, the snow was removed after only one brush stroke for all trials. It is therefore reasonable to conclude that, under identical icing conditions, the aluminum plates behave the same way as the tail blade profiles. Additional testing was conducted with profiles coated with ski wax and Nusil and then compared with the aluminum reference plates. Under the precipitation conditions studied, the Swix wax obtained comparable results, including de-icing with the same tools and the same number of passes. A considerable difference, however, was noticed with Nusil. The latter coating applied to the profile required the use of a harder tool. It did not facilitate the de-icing process in certain concave areas or on the leading edge.

The majority of the tests conducted demonstrated that testing on flat plates can reproduce the de-icing process done on a rotorcraft tail profile. However, they also demonstrated the importance of testing in actual conditions on a full-sized aircraft before considering use due to the possible complexities associated with geometries.

Although the results obtained with this procedure are repeatable and provides relevant information regarding the behavior of a material under winter decontamination conditions, some improvements could be made. To ensure a more reliable and repeatable process, the tools could be instrumented to measure the shear stress applied as a function of time for the purpose of classification. However, at the present moment, the proposed procedure is highly representative of the operational techniques currently used.

\subsection{Overall Classification of the Materials}

As the aim of this procedure is to evaluate each material's ability to assist in manual de-icing, their individual results were compilated to obtain an overall ranking. The results used for this ranking are presented in Table 4. As specified in the methodology, the icephobic behaviors were regrouped to produce one rating out of five. The icephobic rating was obtained using the average of their reduction factor as compared to the reference aluminum. The best overall coating regarding the icephobic behavior was Nusil with a rating of 5.0, followed by SurfEllent with 3.0, then Swix with 2.9, and then Wearlon with 1.3. Aluminum was awarded a rating of 0.4 for comparison purposes. The ratings obtained from the MTA using the four targeted conditions were used without any further modification, with an individual maximum score of 20 . 
Table 4. Ranking of tested materials.

\begin{tabular}{cccccc}
\hline & 1: Reference & 2: Nusil & 3: Swix & 4: SurfEllent & 5: Wearlon \\
\hline ARFCAT & 1 & 8.4 & 2.2 & 6.8 & 6 \\
ARFPO & 1 & 12.5 & 10.7 & 8.9 & 1.1 \\
RF Freezing delay & 1 & 15.7 & 8.3 & 6.6 & 2.6 \\
RF Average & 1 & 12.2 & 7.1 & 7.4 & 3.2 \\
Icephobic Rating (/5) & 0.4 & 5.0 & 2.9 & 3.0 & 1.3 \\
MTA HZR & 0 & 6 & 10 & 1 & 0 \\
MTA LZR & 0 & 6 & Not tested & 9 & 9 \\
MTA Dry SNW & 20 & 14 & 20 & 20 & 15 \\
MTA Adh SNW & 5 & 5 & 10 & 10 & 8 \\
Total & 25.4 & 36.0 & 42.9 & 43.0 & 33.3 \\
Ranking & 5 & 3 & 2 & 1 & 4 \\
\hline
\end{tabular}

The final results for each material were given a score out of 85 . The criterion that stipulates if a material can assist in a manual de-icing is to obtain an overall result higher than that of the aluminum reference. Given this criterion, all tested materials could assist the manual de-icing since each of them received a score higher than that of aluminum, i.e., greater than 25.4. The material with the best performance was found to be SurfEllent with a score of 43.0, followed by Swix with 42.9. The slight difference between the two materials is due to the poor results obtained in the CAT for Swix where the ice had significantly more adherence. The third ranked material was Nusil, even though it demonstrated increased icephobic behavior, it reacted negatively under snow conditions. Finally, the last ranked material was Wearlon, which performed somewhat like aluminum in some conditions, for example, in the MTA high-intensity freezing rain.

From these results, it is important to note that, although a material may show promising results using the CAT or $\mathrm{PO}$, it is not inherent that it will behave the same way when subjected to different icing or snowing precipitation. CAT and $\mathrm{PO}$ are good predictors; however, other icing conditions need to be evaluated before claiming a material to be icephobic.

It is important to note that other significant factors could be integrated within the evaluation process throughout this procedure. One important factor that could be added is the evaluation of the coating's mechanical resistance, especially if the targeted application is in aerospace for aircrafts or rotorcrafts. The critical surfaces of these aircrafts are subjected to several inclement conditions such as rain and sand. It is important to recognize that ice can also undermine the integrity of a coating and significantly reduce its efficiency. Erosion that occurs during normal operation, especially to leading edges, is a serious issue that needs to be evaluated prior to large-scale implementation. Standard tests that are documented and well known to the industry could be used and given a certain weighted percentage within the procedure. On the other hand, a pass/fail criterion could be implemented depending on its criticality.

A second factor that could be added to this analysis is a weighted score reflecting the ease of application. Treating already deployed rotorcrafts with a new material like a coating could be easily done if the method does not require multiple layers or pre-treatments.

Finally, this procedure can be modified to better reflect the needs of the end-user by allocating more weight to a characteristic that is deemed significant for the targeted field of application. Using our classification as an example, if the icing condition deemed to be the most relevant is light-intensity freezing rain and snow is no longer considered to be significant, by increasing the weighted score of LZR to 50 and decreasing all others to 10, the final result would indicate that Nusil should be considered as the coating of choice. However, if the opposite is to be assessed, then more weight should be given to adhering snow, which will ultimately result in Nusil as the least-favorable coating.

When a coating is developed for a targeted application, industrials and academics need to assess if their materials are efficient or at the very least have the capability to optimize their properties. The presented procedure could then be used as a development tool by subjecting materials to the proposed controlled conditions. The main objective 
is to obtain the most efficient materials with tailored performance properties. However, the use of these tools with the proposed conditions and tests may be adapted to ensure that the materials are accurately evaluated. Finally, the most promising materials could be assessed in a full-scale trial with an actual operational setup, i.e., a rotorcraft, to validate the laboratory results. This testing should focus on identifying the vulnerabilities of the coating's performance in the context of an actual rotorcraft operation. This can be done either outdoors in natural icing conditions, or in an appropriately sized icing laboratory capable of accommodating a full-sized aircraft.

\section{Conclusions}

The aim of this paper was to propose a detailed comparative procedure to assess the ability of passive ice protection materials in assisting the manual de-icing of a rotorcraft. The proposed procedure consisted of the characterization of materials under several laboratory tests in order to determine their characteristics of wettability and their icephobic behavior. Finally, their assessment under a multi-tool analysis was conducted to evaluate if they can assist in the manual de-icing, which includes conditions resembling operational reality. The multi-tool analysis allowed for the classification of materials depending on which tools were used, the number of passes necessary, and different atmospheric icing conditions. The comparison scale used five tools that are commercially available. The four icing conditions at $-10{ }^{\circ} \mathrm{C}$ simulated in the laboratory included the following: high-intensity freezing rain, low-intensity freezing rain, dry snow, and partially melted and refrozen snow. Five different materials were used to evaluate the proposed procedure: aluminum, as a reference; two silicone-based coatings, Nusil and SurfEllent; an epoxy-based coating Wearlon; and finally a commercial ski wax, Swix.

The wettability characteristics, which were determined for all materials tested, provided valuable insight into their icephobic behavior. All materials tested were more hydrophobic than aluminum. The material with the most significant icephobic behavior was the Nusil silicone coating with an average reduction factor of 12.2. This was determined by considering the adhesion reduction factor of the CAT, the PO and the reduction factor in freezing delay. The following coatings, SurfEllent, Swix, and Wearlon, also showed some icephobic behavior, however, to a lesser extent, with average reduction factors of 7.4, 7.1, and 3.2, respectively. By evaluating the materials with the MTA, all materials behaved in a different manner. Although Nusil appeared to be promising under ice adhesion, it performed poorly under snow conditions. On the other hand, SurfEllent and Swix received high scores by clearly assisting the manual de-icing in most conditions. Overall, the SurfEllent coating received the highest score, indicating that it could assist the manual de-icing in these targeted conditions.

The overall results show that it is important to undertake several tests including different ice and snow precipitation before concluding a material to be icephobic. On the other hand, having such a procedure, including predictor tests and real operational techniques, certainly allows for a better characterisation of the material(s).

This procedure could easily be adapted to different fields of application by varying the type of frozen precipitation, the type of tools, and even the weight percentage of the results to adequately assess the efficiency of the materials in question. The improvement of the MTA could be undertaken by further instrumenting or automating the process, ensuring repeatability. Finally, this procedure could also be used as a development tool for the optimization and assessment of new materials.

Author Contributions: Conceptualization, J.-D.B., D.P., S.S., M.R. and G.M.; methodology, J.-D.B., D.P. and M.R.; validation, J.-D.B. and G.M.; formal analysis, J.-D.B.; resources, D.P., S.S. and M.R.; writing-original draft preparation, J.-D.B., D.P. and S.S.; writing-review and editing, M.R. and G.M.; supervision, G.M.; project administration, G.M.; funding acquisition, J.-D.B. and G.M. All authors have read and agreed to the published version of the manuscript. 
Funding: The authors would like to thank the Department of National Defense of Canada for funding the research under contract W7714-217494.

Conflicts of Interest: The authors declare no conflict of interest.

\section{References}

1. Ryerson, C.; Gilligan, T.; Koenig, G. Evaluation of three helicopter preflight deicing techniques. In Proceedings of the 37th Aerospace Sciences Meeting and Exhibit, Reno, NV, USA, 11-14 January 1999; p. 499.

2. Gent, R.W.; Dart, N.P.; Cansdale, J.T. Aircraft icing. Philos. Trans. R. Soc. A 2000, 358, 2873-2911. [CrossRef]

3. Brouwers, E.W.; Peterson, A.A.; Palacios, J.L.; Centolanza, L.R. Ice Adhesion Strenght Measurement for Rotor Blade Leading Edge Materials. In Proceedings of the 67th Annual Forum, Virginia Beach, VA, USA, 3-5 May 2011.

4. Kim, J.; Sankar, L.; Palacios, J.; Kreeger, R.E. Numerical and experimental studies of rotorcraft icing phenomena. In Proceedings of the 41st European Rotorcraft Forum 2015, Munich, Germany, 1-4 September 2015.

5. Liu, Y.; Li, L.; Ning, Z.; Tian, W.; Hu, H. Experimental investigation on the dynamic icing process over a rotating propeller model. J. Propuls. Power 2018, 34, 933-946. [CrossRef]

6. Palacios, J.L.; Han, Y.; Brouwers, E.W.; Smith, E.C. Icing environment rotor test stand liquid water content measurement procedures and ice shape correlation. J. Am. Helicopter Soc. 2012, 57, 29-40. [CrossRef]

7. Villeneuve, E.; Volat, C.; Ghinet, S. Numerical and Experimental Investigation of the Design of a Piezoelectric De-Icing System for Small Rotorcraft Part 1/3: Development of a Flat Plate Numerical Model with Experimental Validation. Aerospace 2020, 7, 62. [CrossRef]

8. Wang, Z.; Zhu, C.; Zhao, N. Experimental Study on the Effect of Different Parameters on Rotor Blade Icing in a Cold Chamber. Appl. Sci. 2020, 10, 5884. [CrossRef]

9. Palacios, J.L.; Smith, E.C.; Gao, H.; Joseph, L.R. Ultrasonic Shear Wave Anti-Icing System for Helicopter Rotor Blades. In Proceedings of the Annual Forum Proceeding-AHS International 62nd Annual Forum, Phoenix, AZ, USA, 9-11 May 2006; pp. 1492-1502.

10. Palacios, J.; Wolfe, D.; Bailey, M.; Szefi, J. Ice testing of a centrifugally powered pneumatic deicing system for helicopter rotor blades. J. Am. Helicopter Soc. 2015, 60, 1-12. [CrossRef]

11. Drury, M.D.; Szefi, J.T.; Palacios, J.L. Full-scale testing of a centrifugally powered pneumatic de-icing system for helicopter rotor blades. J. Aircr. 2017, 54, 220-228. [CrossRef]

12. Budinger, M.; Pommier-Budinger, V.; Reysset, A.; Palanque, V. Electromechanical Resonant Ice Protection Systems: Energetic and Power Considerations. AIAA J. 2021, 59, 1-13. [CrossRef]

13. Farzaneh, M.; Jakl, F. Coatings for Protecting Overhead Power Network Equipment in Winter Conditions: Working Group B2. 44; Cigré; 2015. Available online: https:/ / e-cigre.org/publication/631-coatings-for-protecting-overhead-power-network-equipment--inwinter-conditions (accessed on 1 December 2021).

14. Vazirinasab, E.; Maghsoudi, K.; Jafari, R.; Momen, G. A comparative study of the icephobic and self-cleaning properties of Teflon materials having different surface morphologies. J. Mater. Process. Technol. 2020, 276, 116415. [CrossRef]

15. Yancheshme, A.A.; Allahdini, A.; Maghsoudi, K.; Jafari, R.; Momen, G. Potential anti-icing applications of encapsulated phase change material-embedded coatings; a review. J. Energy Storage 2020, 31, 101638. [CrossRef]

16. Yancheshme, A.A.; Momen, G.; Aminabadi, R.J. Mechanisms of ice formation and propagation on superhydrophobic surfaces: A review. Adv. Colloid Interface Sci. 2020, 279, 102155. [CrossRef] [PubMed]

17. Jung, S.; Dorrestijn, M.; Raps, D.; Das, A.; Megaridis, C.M.; Poulikakos, D. Are superhydrophobic surfaces best for icephobicity? Langmuir 2011, 27, 3059-3066. [CrossRef]

18. Zhu, T.; Cheng, Y.; Huang, J.; Xiong, J.; Ge, M.; Mao, J.; Liu, Z.; Dong, X.; Chen, Z.; Lai, Y. A transparent superhydrophobic coating with mechanochemical robustness for anti-icing, photocatalysis and self-cleaning. Chem. Eng. J. 2020, 399, 125746. [CrossRef]

19. Jamil, M.I.; Zhan, X.; Chen, F.; Cheng, D.; Zhang, Q. Durable and scalable candle soot icephobic coating with nucleation and fracture mechanism. ACS Appl. Mater. Interfaces 2019, 11, 31532-31542. [CrossRef] [PubMed]

20. Saito, H.; Takai, K.; Yamauchi, G. Water-and ice-repellent coatings. Surf. Coat. Int. 1997, 80, 168-171. [CrossRef]

21. Brouwers., E.W.; Palacios, J.L.; Smith, E.C. The experimental Investigation of A Rotor Hover Icing Model with Shedding. In Proceedings of the American Helicopter Society 66th Annual Forum, Phoenix, AZ, USA, 11-13 May 2010.

22. Laforte, C.; Laforte, J.L. How a Solid Coating Can Reduce the Adherence of Ice on a Structure. In Proceedings of the Aircraft Ground De-Icing, July 2001. Available online: https://www.researchgate.net/publication/228473956_How_a_solid_coating_ can_reduce_the_adhesion_of_ice_on_a_structure (accessed on 1 December 2021).

23. Tarquini, S.; Antonini, C.; Amirfazli, A.; Marengo, M.; Palacios, J. Investigation of ice shedding properties of superhydrophobic coatings on helicopter blades. Cold Reg. Sci. Technol. 2014, 100, 50-58. [CrossRef]

24. Kimura, S.; Yamagishi, Y.; Sakabe, A.; Adachi, T.; Shimanuki, M. A New Surface Coating for Prevention of Icing on Airfoils; SAE Technical Paper; 2007; pp. 148-7191. Available online: https://www.sae.org/publications/technical-papers/content/2007-01-33 $15 /$ (accessed on 1 December 2021).

25. Karmouch, R.; Coudé, S.; Abel, G.; Ross, G.G. Icephobic PTFE coatings for wind turbines operating in cold climate conditions. In Proceedings of the 2009 IEEE Electrical Power \& Energy Conference (EPEC), Montreal, QC, Canada, 22-23 October 2009; pp. 1-6. 
26. Antonini, C.; Innocenti, M.; Horn, T.; Marengo, M.; Amirfazli, A. Understanding the effect of superhydrophobic coatings on energy reduction in anti-icing systems. Cold Reg. Sci. Technol. 2011, 67, 58-67. [CrossRef]

27. Zhang, S.; Huang, J.; Cheng, Y.; Yang, H.; Chen, Z.; Lai, Y. Bioinspired Surfaces with Superwettability for Anti-Icing and Ice-Phobic Application: Concept, Mechanism, and Design. Small 2017, 13, 1701867. [CrossRef] [PubMed]

28. Shen, Y.; Wu, X.; Tao, J.; Zhu, C.; Lai, Y.; Chen, Z. Icephobic materials: Fundamentals, performance evaluation, and applications. Prog. Mater. Sci. 2019, 103, 509-557. [CrossRef]

29. Zheng, W.; Teng, L.; Lai, Y.; Zhu, T.; Li, S.; Wu, X.; Cai, W.; Chen, Z.; Huang, J. Magnetic responsive and flexible composite superhydrophobic photothermal film for passive anti-icing/active deicing. Chem. Eng. J. 2022, 427, 130922. [CrossRef]

30. Laforte, C.; Blackburn, C.; Perron, J.; Aubert, R. Icephobic Coating Evaluation for Aerospace Application. In Proceedings of the 55th AIAA/ASMe/ASCE/AHS/SC Structures, Structural Dynamics, and Materials Conference, National Harbor, MD, USA, 13-17 January 2014. 